Research Article

\title{
Gliding-Guided Projectile Attitude Tracking Controller Design Based on Improved Adaptive Twisting Sliding Mode Algorithm
}

\author{
Wenguang Zhang and Wenjun Yi \\ National Key Laboratory Transient Physics, Nanjing University of Science and Technology, Nanjing 210094, China \\ Correspondence should be addressed to Wenjun Yi; njustwz@163.com
}

Received 30 January 2020; Revised 18 September 2020; Accepted 1 October 2020; Published 31 October 2020

Academic Editor: Antonio Concilio

Copyright (c) 2020 Wenguang Zhang and Wenjun Yi. This is an open access article distributed under the Creative Commons Attribution License, which permits unrestricted use, distribution, and reproduction in any medium, provided the original work is properly cited.

\begin{abstract}
The finite-time attitude tracking control for gliding-guided projectile with unmatched and matched disturbance is investigated. An adaptive variable observer is used to provide estimation for the unmeasured state which contains unmatched disturbance. Then, an improved adaptive twisting sliding mode algorithm is proposed to compensate for the matched disturbance dynamically with better transient quality. Finally, a proof of the finite-time convergence of the closed-loop system under the disturbance observer and the adaptive twisting sliding mode-based controller is derived using the Lyapunov technique. This attitude tracking control scheme does not require any information on the bounds of uncertainties. Simulation results demonstrate that the proposed method which is able to acquire the minimum possible values of the control gains guaranteeing the finite-time convergence performs well in chattering attenuation and tracking precision.
\end{abstract}

\section{Introduction}

The most significant difference between gliding-guided projectile (GGP) and traditional projectile is that the former one is equipped with wing assembly. On the one hand, the wing assembly makes the projectile be capable of gliding, which enables the gliding-guided projectile to strike the target from a greater distance. On the other hand, the wing assembly provides the projectile with the ability of controlling its flight path, which means that the gliding-guided projectile can achieve precise strikes $[1,2]$. Due to these advantages, the gliding-guided projectile has become the current research hotspot. It should be noted that the main way to ensure the stability of a projectile's airframe is a high body roll-axis spin rate, which is a significant difference from traditional missiles, and is the source of nonlinear coupling between the yaw and pitch channel of GGP. Therefore, the design of GGP, especially the design of the attitude controller, is a challenging task. This is due to the fact that the GGP model suffers from high nonlinearity and strong coupling characteristics. In addition, a GGP is vulnerable to various disturbances during flight, such as the aerodynamic parameter perturbation, the unmodeled dynamics, and strong and sudden wind gusts which change greatly along the latitude and longitude [3].

To compensate for uncertainties and disturbances suffered by the flight control system, a lot of works have been carried out. In [4], the authors propose a pitch attitude and lateral decoupling control based on active disturbance rejection control for aircraft. To obtain robustness, they utilize an extended state observer (ESO). In [5], the finite-time attitude control for a reusable launch vehicle with unmatched disturbance is investigated, and they propose an adaptive multivariable disturbance compensation method to estimate the disturbance. In [6], the adaptive control on the longitudinal dynamics of a hypersonic flight vehicle in the presence of wind effects is investigated. The unknown disturbance due to wind is estimated by neural networks. In [7], they propose a dual closed-loop control 
framework for attitude control of a quadrotor. In the dualloop framework they proposed, active disturbance rejection control and proportional-derivative control are used in the inner and outer loops, respectively. To cancel the effect of dynamic disturbance, they use an ESO. In [8], they utilize the prescribed performance control technique to tackle the issue of attitude control of hypersonic flight vehicles (HFVs), which largely improves the transient characteristics of HFVs. In [9], the authors propose an intelligent flight control scheme based on reinforcement learning technique. Besides, many other control methods, such as fuzzy control, neural network control, backstepping control, and model following control, have been applied on this issue [10-20]. It should be noted that various feedback linearizations have been applied to this issue [21-23]. The aims of such methods are to cancel the nonlinear dynamics via mathematical transformation so as to control the system in a linear manner. It is able to provide a full envelope nonlinear flight control system. However, the design techniques need much information about the system; otherwise, the output will not be desired.

Higher-order sliding mode (HOSM) control is another popular method to deal with an uncertain system. A twisting controller (TC) is historically the first 2-SMC algorithm [24] that drives the output and derivative of the system to the origin in finite time in the presence of disturbance whose boundary is known. If the boundary of the disturbance is unknown, it is necessary to design adaptive twisting sliding mode control (ATSMC) methods to satisfy the condition for sliding mode to exist. One efficient scheme is designing algorithms which are able to adjust dynamically the control gains. In [25], this kind of adaptive twisting sliding mode control is integrated into attitude tracking controller for the reentry reusable launch vehicle. In [26], the twisting sliding mode control law is modified with a proposed gain adaptation strategy to improve the attitude tracking performance of quadrotor unmanned aerial vehicles. In [27], the Lyapunov theory is used to design the ATSMC. However, the aforementioned methods fail to search the minimum possible value of the control gain. As we know, the amplitude of chattering which hinders the applications of the sliding mode control is proportional to the magnitude of discontinuous control.

In [28], an adaptive algorithm is designed via equivalent control. Their method is able to search the minimum possible value of control. In their work, the gains can be adjusted adaptively to compensate for the disturbances with lower values without knowledge of the bounds of the disturbances. However, this method may lead to large chattering when the values of the gains are close to the ideal ones. This is due to the fact that the changing rate of the gains still keeps a large value in such circumstances.

Motivated by the aforementioned work, we propose a robust attitude tracking scheme for GGP with chattering attenuation in this paper. The error dynamics of the attitude tracking system suffers from both matched and unmatched disturbances, where the bounds of disturbances are not known. In order to estimate the unmeasured state influenced by unmatched disturbance, an adaptive observer is utilized. Then, an improved equivalent control-based adaptive twisting sliding mode control (IECATSMC) algorithm for [28] is designed and adopted by the controller to compensate for the matched disturbance dynamically. The main contributions of this paper can be summarized as follows:

(i) The improved adaption algorithm is able to adaptively adjust the changing rates of the gains on a larger scale. Thus, the transient characteristics of the adaptation strategy in [28] are improved. Since the proposed control scheme is able to acquire the minimum possible values of the control gains, it performs better in chattering attenuation than the fixed gain TSMC and the existing ATSMC, which can be seen from the simulation results given in the latter

(ii) A high-performance disturbance estimator proposed in [29] is used in this paper to reconstruct the unmeasurable system state, which extends the application of this estimator. More importantly, by doing so, the finite-time convergence property of the closed-loop system is guaranteed

(iii) A proof of the finite-time convergence of the closedloop system under the observer and the IECATSMC-based controller is derived using the Lyapunov stability theory

The paper is organized as follows: the GGP attitude kinematics model is given in Section 2; then, error dynamics of attitude tracking system is derived. To estimate the unmeasured state in error dynamics, an adaptive observer is utilized in Section 3. The improved adaptive twisting algorithm which is able to search the minimum possible values of the control gains is investigated for the attitude control in Section 4, and a proof of the finite-time convergence for the overall system is derived. The simulation results are provided in Section 5. Finally, concluding remarks are summarized in Section 6.

Notations. Throughout the paper the following notations will be used. For a given vector $\mathbf{x}=\left[x_{1}, \cdots, x_{i}, \cdots, x_{n}\right]^{T} \in \mathfrak{R}$, denote $\|\mathbf{x}\|=\sqrt{\mathbf{x}^{T} \mathbf{x}}$. For a given scalar variable $y$, denote $|y|$ as the absolute value of $y$.

\section{Gliding-Guided Projectile Attitude Kinematics Model}

In actual flight, the speed of the GGP is variable due to the varying thrust and drag. However, if we study the dynamics of the projectile which is gliding along a very short flight path, we can safely think that the flight speed and rotational speed of the projectile are constant. Besides, we can also neglect the variation of the dynamic pressure and Mach number as well as other physical parameters. Then, under the assumption that the attack angle and side slip angle are small, the attitude kinematics model of the gliding-guided projectile can be constructed as $[30,31]$ 


$$
\left\{\begin{array}{l}
\dot{\theta}=\alpha_{1} \alpha-\alpha_{2} \beta, \\
-\dot{\varphi} \cos \theta=\alpha_{1} \beta-\alpha_{2} \alpha, \\
\ddot{\vartheta}=b_{1} \alpha-b_{2} \beta+b_{4} \delta_{z}-\alpha_{3} \delta_{y}-b_{3} \dot{\vartheta}+b_{5} \cos \vartheta \cdot \psi-b_{5} \dot{\vartheta} \\
\ddot{\psi} \cos \vartheta=b_{1} \beta+b_{2} \alpha+b_{4} \delta_{y}-\alpha_{3} \delta_{z}-b_{3} \cos \vartheta \cdot \dot{\psi}-b_{5} \dot{\vartheta}
\end{array}\right.
$$

where $a_{i}$ and $b_{i}$ denote kinetic coefficients, where $a_{1}=$ $(Q S / m v)\left(C_{L}^{\alpha}+C_{L}^{\delta}\right), \quad a_{2}=(Q S / m v) C_{\mu}^{\prime \prime}(\dot{\gamma} d / v), \quad a_{3}=(Q S / m v)$ $C_{L}^{\delta}, b_{1}=(Q S / E)\left(m_{z}^{\prime}+m_{\sigma}^{\prime}\right), b_{2}=((Q S l \dot{\gamma} D) / E v) m_{p}^{\prime} b_{3}=(Q S l D /$ $E v) m_{z z}^{\prime}, b_{4}=(Q S l / E) m_{\sigma}^{\prime}$, and $b_{5}=C \dot{\gamma} / E$.

By accepting that the attitude change speed is more faster than the change speed of the velocity vector direction of the projectile center of mass on the short flight path [30], and without loss of generity, we assume that $\dot{\vartheta} \approx \dot{\alpha}, \dot{\varphi} \approx \dot{\beta}$. From (2) and the assumption of $\alpha=\vartheta-\theta, \beta=(\psi-\varphi) \cos \theta$, and $\cos \theta \approx \cos \vartheta$, we obtain

$$
\left\{\begin{array}{l}
\dot{\alpha}=\dot{\vartheta}-a_{1} \alpha+a_{2} \beta, \\
\dot{\beta}=(\dot{\psi}-\dot{\varphi}) \cos \vartheta+(\psi-\varphi) \dot{\vartheta} \sin \vartheta \\
\frac{d(\vartheta-\theta)}{d t^{2}}=-k_{1} \dot{\alpha}-k_{2} \dot{\beta}+k_{3} \alpha-k_{4} \beta+ \\
k_{5} \delta_{z}-k_{6} \delta_{y}, \\
\frac{d((\psi-\varphi) \cos \theta)}{d t^{2}}=-k_{1} \dot{\beta}-k_{2} \dot{\alpha}+k_{3} \beta+k_{4} \alpha \\
+k_{5} \delta_{y}-k_{6} \delta_{z},
\end{array}\right.
$$

where $k_{1}=b_{3}+a_{1}, k_{2}=b_{5}+a_{2}, k_{3}=b_{1}+b_{3} a_{1}+b_{5} a_{2}, k_{4}=$ $b_{2}-b_{3} a_{2}-b_{5} a_{1}, k_{5}=b_{4}$, and $k_{6}=a_{3}$.

Assume that the steering gear system of the projectile is designed as a second-order one. For the convenience of study, we only consider the steady output of equivalent rudder deflection [32]:

$$
\left[\begin{array}{l}
\delta_{z} \\
\delta_{y}
\end{array}\right]=k_{s} k_{r}\left[\begin{array}{cc}
\cos \gamma_{d} & \sin \gamma_{d} \\
-\sin \gamma_{d} & \cos \gamma_{d}
\end{array}\right]\left[\begin{array}{l}
\delta_{z c} \\
\delta_{y c}
\end{array}\right],
$$

where

$$
\left\{\begin{array}{l}
k_{s}=\frac{1}{\sqrt{\left(1-T_{s}^{2} \dot{\gamma}^{2}\right)^{2}+\left(2 \mu_{s} T_{s} \dot{\gamma}\right)^{2}}}, \\
k_{r}=\tau_{d} \dot{\gamma}+\arccos \frac{1-T_{s}^{2} \dot{\gamma}^{2}}{\sqrt{\left(1-T_{s}^{2} \dot{\gamma}^{2}\right)^{2}+\left(2 \mu_{s} T_{s} \dot{\gamma}\right)^{2}}},
\end{array}\right.
$$

where $\delta_{y c}$ and $\delta_{z c}$ stand for equivalent rudder deflect angle control commands for pitching channel and yaw channel, respectively; $k_{s}$ is the gain of canard; $T_{s}$ denotes the time constant of steer gear system; $\mu_{s}$ denotes the system damping ratio; $\tau_{d}$ represents delay time; , and $\gamma_{d}$ represents delay phase angle.

Taking matched and unmatched disturbances into consideration, from $((1))$ and $((2))$, we get

$$
\left\{\begin{array}{l}
\dot{\alpha}=\dot{\vartheta}-a_{1} \alpha+a_{2} \beta+d_{1}, \\
\dot{\beta}=(\dot{\psi}-\dot{\varphi}) \cos \vartheta+(\psi-\varphi) \dot{\vartheta} \sin \vartheta+d_{2} \\
\frac{d(\vartheta-\theta)}{d t^{2}}=f_{1}+\Delta_{f 1}+A_{1} \Theta^{T}+ \\
\left(\mathbf{B}_{1}+\Delta_{B_{1}}\right) U+\dot{d}_{1}, \\
\frac{d((\psi-\varphi) \cos \theta)}{d t^{2}}=f_{2}+\Delta_{f_{2}}+A_{2} \Theta^{T}+\left(\mathbf{B}_{2}+\Delta_{B_{2}}\right) U+\dot{d}_{2},
\end{array}\right.
$$

where

$$
\begin{aligned}
& \boldsymbol{\Theta}=\left[\begin{array}{ll}
\alpha & \beta
\end{array}\right]^{T}, \\
& \mathbf{U}=\left[\begin{array}{ll}
\delta_{y c}, \delta_{z c}
\end{array}\right]^{T}, \\
& f_{1}=-k_{1} \dot{\alpha}-k_{2} \dot{\beta}+k_{3} \alpha-k_{4} \beta, \\
& f_{2}=-k_{1} \dot{\beta}-k_{2} \dot{\alpha}+k_{3} \beta+k_{4} \alpha, \\
& \mathbf{A}_{1}=\left[\begin{array}{ll}
k_{3} & -k_{4}
\end{array}\right]^{T}, \\
& \mathbf{A}_{2}=\left[\begin{array}{ll}
k_{4} & k_{3}
\end{array}\right]^{T}, \\
& \mathbf{B}_{1}=\left[\begin{array}{ll}
\kappa_{6} & \kappa_{5}
\end{array}\right]^{T}, \\
& \mathbf{B}_{2}=\left[\begin{array}{ll}
\kappa_{5} & -\kappa_{6}
\end{array}\right]^{T}, \\
& \kappa_{5}=k_{5} k_{s} k_{r} \cos \gamma_{d}-k_{6} k_{s} k_{\mathrm{r}} \sin \gamma_{d}, \\
& \kappa_{6}=k_{5} k_{s} k_{r} \sin \gamma_{d}+k_{6} k_{s} k_{r} \cos \gamma_{d},
\end{aligned}
$$

besides, $\Delta_{f_{1}}, \Delta_{f_{2}}, \Delta_{B_{1}}$, and $\Delta_{B_{2}}$ are uncertainties induced by parameter variation and model simplification; $d_{1}, d_{2}$ stand for external unmeasurable disturbances.

The object is to design a control input $\mathbf{U}=\left[\delta_{y c}, \delta_{z c}\right]^{T}$ so as to drive the attitude angle vector $\Theta$ to follow the control command $\boldsymbol{\Theta}_{c}=\left[\alpha_{c}, \beta_{c}\right]^{T}$ in spite of the internal and external disturbances.

Thus, defining the attitude tracking error as $\mathbf{e}_{\boldsymbol{\Theta}}=\boldsymbol{\Theta}-\boldsymbol{\Theta}_{c}$, using (1) and recalling the relationships $\alpha=\vartheta-\theta$ and $\beta=$ $(\psi-\varphi) \cos \theta$, the error dynamics for (5) can be calculated as

$$
\left\{\begin{array}{l}
\dot{\mathbf{e}}_{\Theta}=\mathbf{z}_{\Theta}, \\
\dot{\mathbf{z}}_{\Theta}=\mathbf{F}+\mathbf{A} \Theta+\mathbf{B U}-\ddot{\Theta}_{c}+\Delta,
\end{array}\right.
$$


where

$$
\begin{aligned}
\mathbf{z}_{\boldsymbol{\Theta}} & =\left[\begin{array}{c}
\dot{\vartheta}-a_{1} \alpha+a_{2} \beta \\
(\dot{\psi}-\dot{\varphi}) \cos \vartheta+(\psi-\varphi) \dot{\vartheta} \sin \vartheta
\end{array}\right]-\dot{\boldsymbol{\Theta}}_{c}+\Delta_{1}, \\
\boldsymbol{\Delta}_{1} & =\left[\begin{array}{ll}
d_{1} & d_{2}
\end{array}\right]^{T} \\
\mathbf{F} & =\left[\begin{array}{ll}
f_{1} & f_{2}
\end{array}\right]^{T} \\
\mathbf{A} & =\left[\begin{array}{ll}
\mathbf{A}_{1} & \mathbf{A}_{2}
\end{array}\right]^{T}, \\
\mathbf{B} & =\left[\begin{array}{ll}
\mathbf{B}_{1} & \mathbf{B}_{2}
\end{array}\right]^{T}, \\
\boldsymbol{\Delta} & =\left[\begin{array}{ll}
\Delta_{f_{1}}+\Delta_{U_{1}} \\
\Delta_{f_{2}}+\Delta_{U_{2}}
\end{array}\right]+\dot{\Delta}_{1}
\end{aligned}
$$

where the deviations $\Delta_{U_{1}}=\Delta_{B_{1}} \mathbf{U}, \Delta_{U_{2}}=\Delta_{B_{2}} \mathbf{U}$ induced by the uncertainties in $B_{1}$ and $B_{2}$ can be seen as internal disturbances.

The following assumption adopted from [29] is needed to the existences of causal control schemes for system (5).

Assumption 1. The disturbances $\Delta_{f_{1}}, \Delta_{f_{2}}, \boldsymbol{\Delta}_{B_{1}}, \Delta_{B_{2}}, d_{1}$, and $d_{2}$ and the first-order derivatives of them are bounded, but the boundaries are unknown.

\section{Finite-Time Adaptive Observer for $z_{\Theta}$}

As we see from (6), the error state contains unknown disturbance $\Delta_{1}$; therefore, it cannot be measured directly. To addresss this problem, an adaptive finite-time variable observer is used for (5) in this section.

Assumption 2. Suppose that the disturbance $\|\boldsymbol{\Delta}\| \leq C$ and $\|\dot{\Delta}\| \leq C^{\prime}$, where $C$ and $C^{\prime}$ are unknown.

For system (5), the controller based on twisting algorithm can be designed as

$$
\mathbf{U}=\frac{1}{\mathbf{B}}\left(-\mathbf{F}-\mathbf{A} \Theta-\ddot{\Theta}_{c}-r_{1} \frac{\mathbf{e}_{\Theta}}{\left\|\mathbf{e}_{\Theta}\right\|}-r_{2} \frac{\mathbf{z}_{\Theta}}{\left\|\mathbf{z}_{\Theta}\right\|}\right),
$$

where $r_{1}, r_{2}>0$ are control gains. It should be noted that the contoller (16) is impractical because of the existence of lumped disturbance $\Delta$, whose upper bound is unknown. Meanwhile, as mentioned before, the error state $\mathbf{z}_{\Theta}$ is not measurable, so an adaptive finite-time observer is needed. Inspired by [29, 33], an adaptive finite-time observer for (16) is formulated as

$$
\dot{\omega}_{1}=-k_{\Gamma_{1}} \frac{\mathbf{s}_{\Gamma}}{\left\|\mathbf{s}_{\Gamma}\right\|^{1 / 2}}+\omega_{2},
$$

$$
\dot{\omega}_{2}=-k_{\Gamma_{2}} \frac{\mathbf{s}_{\Gamma}}{\left\|\mathbf{s}_{\Gamma}\right\|},
$$

where the auxiliary sliding variable

$$
\mathbf{s}_{\Gamma}=\varpi_{1}-\mathbf{e}_{\Theta}
$$

and the adaptive gains $k_{\Gamma_{1}}$ and $k_{\Gamma_{2}}$ are

$$
\left\{\begin{array}{l}
\dot{k}_{\Gamma_{1}}= \begin{cases}\omega_{1} \sqrt{\frac{\iota_{1}}{2},} & \text { if }\left\|\mathbf{s}_{\Gamma}\right\|>\sigma_{T}, \\
0, & \text { if }\left\|\mathbf{s}_{\Gamma}\right\| \leq \sigma_{T},\end{cases} \\
k_{\Gamma_{2}}=\varepsilon_{1} k_{\Gamma_{1}},
\end{array}\right.
$$

where $\sigma_{T}$ represents the arbitrary small threshold value, and $\omega_{1}, \iota_{1}$, and $\varepsilon_{1}$ are positive constants. It can be concluded that $\mathbf{z}_{\Theta}$ can be estimated by the term $\omega_{2}$ in finite time regardless of the unknown bounded uncertainty $\Delta[29]$.

\section{Main Results}

If $r_{1}$ and $r_{2}$ in (16) satisfy $r_{1}>2 C$ and $r_{2}>C$, the system represented by (5) is stable [34]. However, to make traditional twisting sliding mode control strategies meet the demand is a challenging thing because the upper bound of disturbance is often unknown especially in the case of gliding-guided projectile. Therefore, adaptive methods which can adjust the values of control gains have been proposed [27]. It should be noted that such methods usually cause large gains, which may result in an undesirable aggressive chattering phenomenon. In this section, an improved adaptive twisting sliding mode control method is developed to search the minimum possible values of control with better transient quality.

Using $\widehat{\mathbf{z}}_{\Theta}$ estimated in (17), the actual adaptive twisting controller is then designed as

$$
\mathbf{U}=\frac{1}{\mathbf{B}}\left(-\mathbf{F}-\mathbf{A} \Theta-\ddot{\boldsymbol{\Theta}}_{c}-r_{1} \frac{\mathbf{e}_{\Theta}}{\left\|\mathbf{e}_{\Theta}\right\|}-r_{2} \frac{\widehat{\mathbf{z}}_{\Theta}}{\left\|\widehat{\mathbf{z}}_{\Theta}\right\|}\right),
$$

where the estimation $\widehat{\mathbf{z}}_{\boldsymbol{\Theta}}=\Phi_{2}=\mathbf{z}_{\boldsymbol{\Theta}}+\mathbf{e}$ with e represents the estimation error of $\mathbf{z}_{\Theta}$. Substituting (25) into (5), the closed-loop system is changed into

$$
\left\{\begin{array}{l}
\dot{\mathbf{e}}_{\Theta}=\widehat{\mathbf{z}}_{\Theta}-\mathbf{e}, \\
\dot{\mathbf{z}}_{\Theta}=-r_{1} \frac{\mathbf{e}_{\Theta}}{\left\|\mathbf{e}_{\Theta}\right\|}-r_{2} \frac{\widehat{\mathbf{z}}_{\Theta}}{\left\|\widehat{\mathbf{z}}_{\Theta}\right\|}+\widehat{\Delta},
\end{array}\right.
$$

where $\widehat{\Delta}=\dot{\mathbf{e}}+\Delta$. 
4.1. Improved Adaptive Twisting Sliding Mode Control Algorithm Design. To simplify the adaptive controller design, the ideal estimate of $\mathbf{z}_{\Theta}$ will be exploited, and the following representation for (26) can be considered:

$$
\left\{\begin{array}{l}
\dot{\mathbf{x}}_{1}=\mathbf{x}_{2}, \\
\dot{\mathbf{x}}_{2}=-h_{1}(t) \frac{\mathbf{x}_{1}}{\left\|\mathbf{x}_{1}\right\|}-h_{2}(t) \frac{\mathbf{x}_{2}}{\left\|\mathbf{x}_{2}\right\|}+\mathbf{f},
\end{array}\right.
$$

where the state variables $\mathbf{x}_{1}, \mathbf{x}_{2} \in \Re^{n}$, and the control gains $h_{1}=L(t), h_{2}=0.5 L(t)$ with $L(t)$ to be designed. f represents lumped uncertainty, which satisfies $\|\mathbf{f}\| \leq D_{0}$ and $\|\dot{\mathbf{f}}\| \leq D_{1}$, where the constants $D_{0}$ and $D_{1}$ are unknown.

Before we proceed, the following lemma is needed to be introduced.

Lemma 3. Considering the system defined as (29), if the gain $L$ satisfies $L>2 D_{0}$, then the states $\mathbf{x}_{1}$ and $\mathbf{x}_{2}$ will converge to origin in finite time.

The proof of Lemma 3 is given in Appendix A.

Thus, the problem to be solved in the following part becomes to design a gain adaptive algorithm, which ensures the condition $L>2 D_{0}$ holds without any knowledge about the disturbance $f$.

Theorem 4. For the uncertain systems (27), the dynamics of the control gains are designed as $h_{1}=L(t), h_{2}=0.5 L(t)$, where $L(t)$ is defined as

$$
\begin{aligned}
\dot{L} & =e^{\rho(|\xi|-\zeta)} \eta L \text { sign }(\xi)-M\left[L-L^{+}\right]_{+}+M[\mu-L]_{+}, \\
{[z]_{+} } & :=\left\{\begin{array}{lll}
1 & \text { if } & z \geq 0, \\
0 & \text { if } & z<0,
\end{array}\right.
\end{aligned}
$$

where $\rho, \zeta, \eta, M$, and $L^{+}$are positive constants, and they satisfy $M>e^{\rho(|\xi|-\zeta)} \eta L^{+}, L>D_{p 0}$. $\xi$ satisfies

$$
\xi=\left\|\left[\frac{\mathbf{x}_{1}}{\left\|\mathbf{x}_{1}\right\|}+0.5 \frac{\mathbf{x}_{2}}{\left\|\mathbf{x}_{2}\right\|}\right]_{e q}\right\|-\varepsilon,
$$

where $[-]_{e q}$ is called the equivalent control [23] function, which is an average value ranging from $(-1,1) . \varepsilon$ is a positive constant and satisfies $\varepsilon \in(0,0.5)$. Then, the states $\mathbf{x}_{i}$ $(i=1,2)$ will converge to origin in finite time.

Proof. The analysis can be divided into three phases.
Phase 1. the gain $L$ satisfies $L<2 D_{0}$.

The system (28) is on reaching phase, so we have

$$
\left\|\left[\frac{\mathbf{x}_{1}}{\left\|\mathbf{x}_{1}\right\|}-0.5 \frac{\mathbf{x}_{2}}{\left\|\mathbf{x}_{2}\right\|}\right]_{\mathrm{eq}}\right\|>\left\|\frac{\mathbf{x}_{1}}{\left\|\mathbf{x}_{1}\right\|}\right\|-0.5\left\|\frac{\mathbf{x}_{2}}{\left\|\mathbf{x}_{2}\right\|}\right\|=0.5 .
$$

Recall that $\varepsilon \in(0,0.5)$, thus

$$
\xi=0.5-\varepsilon>0,
$$

so the value of $L$ will keep increasing until it is large enough, which means $\int_{t_{0}}^{t_{1}} e^{\rho(|\xi|-\zeta)} \eta L \operatorname{sign}(\xi)-M\left[L-L^{+}\right]^{+}+M$ $[\mu-L]_{+} d t>2 D_{0}$ can be achieved.

Assuming $L<l^{+}$, we can have

$$
\dot{L} \geq \eta L+M[\mu-L]_{+} \geq \eta L
$$

here, $e^{\rho(|\xi|-\zeta)} \geq 1$ is used. Therefore, by solving the inequation $L \leq 2 D_{0}$, we can obtain $t_{1} \geq \ln \left(\left|2 D_{0}-L\left(t_{0}\right)+e^{\eta t_{0}}\right|\right)$, which means the condition $L \leq 2 D_{0}$ can be achieved at most after $\ln \left(\left|2 D_{0}-L\left(t_{0}\right)+e^{\eta t_{0}}\right|\right)$.

Phase 2. the gain $L$ satisfies $L>2 D_{0}$.

According to Lemma 3 , the states $\mathbf{x}_{1}$ and $\mathbf{x}_{2}$ will converge to the origin in finite time. Furthermore, the convergence time $t_{2}$ satisfies $t_{2} \leq t_{0}+t_{1}+\left(16 \bar{\lambda}_{\max }^{3 / 4}(P) V_{0}^{1 / 4}\right.$ $\left.\left(t_{1}\right)\right) / \bar{E}$, where the definitions of $P$ and $\bar{E}$ can be seen in Appendix A.

Phase 3. the system enters the sliding mode.

First, Let us assume that $L \in\left[\mu, L^{+}\right]$, which means $\|\mathbf{f}\| / \varepsilon$ $>\mu$, and the time derivative of $\left[\operatorname{sign}\left(\mathbf{x}_{1}\right)+0.5 \operatorname{sign}\left(\mathbf{x}_{2}\right)\right]_{\mathrm{eq}}$ exist. A Lyapunov function candidate is defined as $V(\xi)=$ $(1 / 2) \xi^{2}$. Since system (27) has entered the sliding mode in phase 3 , the following equation holds

$$
\left\|u_{\mathrm{eq}}\right\|=L\left\|\left[\frac{\mathbf{x}_{1}}{\left\|\mathbf{x}_{1}\right\|}+0.5 \frac{\mathbf{x}_{2}}{\left\|\mathbf{x}_{2}\right\|}\right]_{\mathrm{eq}}\right\|=\|\mathbf{f}\|,
$$

where $u_{\text {eq }}=L\left[\left(\mathbf{x}_{1} /\left\|\mathbf{x}_{1}\right\|\right)+0.5\left(\mathbf{x}_{2} /\left\|\mathbf{x}_{2}\right\|\right)\right]_{\mathrm{eq}}$ is defined as equivalent control input. Taking the time derivative of 


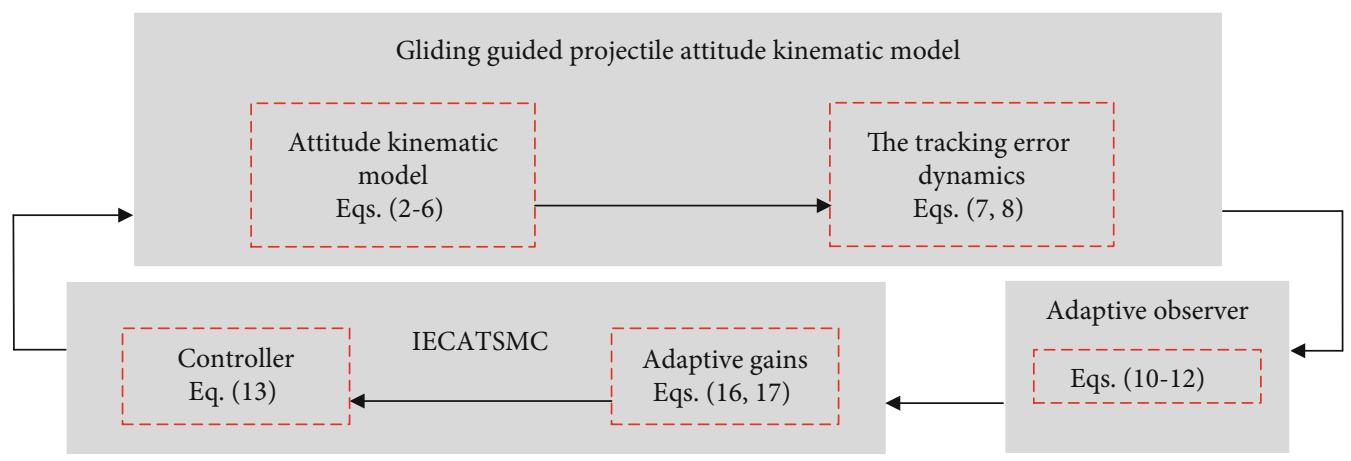

FIGURE 1: Schematic diagram of the proposed control strategy.

$V(\xi)$, assuming that $L(t) \in\left[\mu, L^{+}\right]$, which means that $\|\mathbf{f}\| /$ $\varepsilon>\mu$ and using (28), (29), and (33) yield

$$
\begin{aligned}
\dot{V}_{0}(\xi)= & \dot{\xi} \xi=\xi \frac{d}{d t}\left\|\left[\frac{\mathbf{x}_{\mathbf{1}}}{\left\|\mathbf{x}_{\mathbf{1}}\right\|}+0.5 \frac{\mathbf{x}_{\mathbf{2}}}{\left\|\mathbf{x}_{\mathbf{2}}\right\|}\right]_{\mathrm{eq}}\right\|=\xi \frac{d}{d t}\left(\frac{\|\mathbf{f}\|}{L}\right) \\
= & -\|\mathbf{f}\| \xi L^{-2} \dot{L}+\xi L^{-1} \frac{d}{d t}(\|\mathbf{f}\|) \\
= & -\|\mathbf{f}\| \xi L^{-2}\left(e^{\rho(|\xi|-\zeta)} \eta L \operatorname{sign}(\xi)-M-\left[L-L^{+}\right]_{+}\right) \\
& \left.+M[\mu-L]_{+}\right)+\xi L^{-1} \mathbf{f}^{T} \dot{\mathbf{f}}\|\mathbf{f}\|^{-1} \\
\leq & -\varepsilon e^{\rho(|\xi|-\zeta)} \eta \mu|\xi| L^{-1} \eta|\xi|+|\xi| L^{-1} D_{1} \\
= & -|\xi| L^{-1}\left(\varepsilon e^{\rho(|\xi|-\zeta)} \eta \mu-D_{1}\right)
\end{aligned}
$$

If $\eta>D_{1} /\left(\varepsilon \mu \mid e^{\rho(|\xi|-\zeta)}\right)$, we will have

$$
\dot{V}(\xi) \leq-\sqrt{2} \frac{\left(\varepsilon e^{\rho(|\xi|-\zeta)} \eta \mu-D_{1}\right.}{L^{+}} \sqrt{V(\xi)} .
$$

Therefore, $V(\xi)=0$ is achievable, at least after $t_{f}=L^{+}$/ $\left(\varepsilon e^{\rho(|\xi|-\zeta)} \eta \mu-D_{1}\right)\left|\xi\left(t_{2}\right)\right|$. After the adaption process for $L$ is over $\left(t>t_{f}\right)$, we obtain

$$
\left\|\left[\frac{\mathbf{x}_{1}}{\left\|\mathbf{x}_{1}\right\|}+0.5 \frac{\mathbf{x}_{2}}{\left\|\mathbf{x}_{2}\right\|}\right]_{\mathrm{eq}}\right\|=\frac{\|\mathbf{f}\|}{L}=\varepsilon
$$

which means $L=\|\mathbf{f}\| / \varepsilon$. Another case is that $(\|\mathbf{f}\|) / \varepsilon>\mu$, then $L$ increases until $L(t)=\mu$, and it will be maintained at that level. The theorem is proven.

If $\varepsilon$ is very close to 0.5 , the minimal possible gain will be found for the current value of disturbance. As a result, the amplitude of chattering will be reduced.
TABle 1: Parameters for a gliding-guided projectile.

\begin{tabular}{lcccc}
\hline$b_{1} / s^{-2}$ & $b_{2} / s^{-2}$ & $b_{3} / s^{-2}$ & $b_{4} / s^{-2}$ & $b_{5} / s^{-2}$ \\
-129.4 & 0 & 0.291 & 90.519 & 1.886 \\
$a_{1} / s^{-1}$ & $a_{2} / s^{-1}$ & $a_{3} / s^{-1}$ & $\dot{\gamma}(r / s)$ & $\tau / s$ \\
0.287 & 0 & 0.136 & 10 & 0.015 \\
$k_{s}$ & $T_{s} / s$ & $\mu_{s}$ & $\delta_{\max } /{ }^{o}$ & $\dot{\delta}_{\max } /(\mathrm{rad} / s)$ \\
1 & $1 / 150$ & 0.7 & 20 & 200 \\
\hline
\end{tabular}

Remark 5. The main feature of the improved adaptive algorithm is that it can adaptively adjust the changing rate of the gain $L$, which is realized by a regulatory factor $e^{\rho(|\xi|-\zeta)}$. The factor enables the changing rate of $L$ to be larger or smaller than $\eta$. In general, when $|\xi|$ becomes larger than $\zeta$, which means $L$ is far away from its ideal value, $e^{\rho(|\xi|-\zeta)}$ can also increase and become larger than 1 . Hence, $L$ will tend more quickly toward its ideal value. On the other hand, once $|\xi|$ enters a small region $C_{\xi}=\{|\xi| \leq \zeta\}$, which means $L$ has been already very close to its ideal value, $e^{\rho(|\xi|-\zeta)}$ will become smaller than 1. As a result, the amplitude of gain oscillation will be reduced so that the condition $L>2\|\Delta\|$ can be safely ensured.

Remark 6. The function $\left[\left(\mathbf{x}_{1} /\left\|\mathbf{x}_{1}\right\|\right)+0.5\left(\mathbf{x}_{2} /\left\|\mathbf{x}_{2}\right\|\right)\right]_{\mathrm{eq}}$ can be derived by low pass filter

$$
\tau \dot{\mathbf{z}}_{1}+\mathbf{z}_{1}=\frac{\mathbf{x}_{1}}{\left\|\mathbf{x}_{1}\right\|}+0.5 \frac{\mathbf{x}_{2}}{\left\|\mathbf{x}_{2}\right\|}, \quad \mathbf{z}_{1}=[0,0]^{T}
$$

with a small time constant $\tau>0$, respectively. The output $\mathbf{z}_{1}$ satisfies

$$
\left\|\mathbf{z}_{1}-\left[\frac{\mathbf{x}_{\mathbf{1}}}{\left\|\mathbf{x}_{\mathbf{1}}\right\|}+0.5 \frac{\mathbf{x}_{\mathbf{2}}}{\left\|\mathbf{x}_{\mathbf{2}}\right\|}\right]_{e q}\right\| \leq O(\tau) \underset{\tau \rightarrow 0}{\longrightarrow} 0 .
$$


TABLE 2: Control parameters of the three algorithms.

\begin{tabular}{lc}
\hline Method & Parameters \\
\hline IECATSMC & $\rho=2, \zeta=0.07, \eta=5.59, L^{+}=50, \mu=0.1, M=279.5279, \varepsilon=0.49, \omega_{1}=4.9, \iota_{1}=2.4, \varepsilon_{1}=0.6, \sigma_{T}=10^{-3}$ \\
ATSMC [34] & $L_{\min }=32, \omega_{1}=4.9, \gamma_{1}=1.1, \mu_{1}=0.0004, \chi=40$ \\
CTSMC [24] & $L=20$ \\
\hline
\end{tabular}

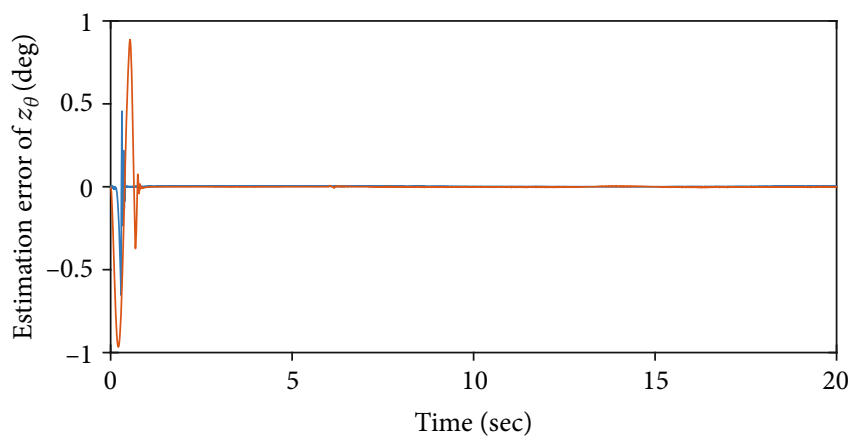

(a)

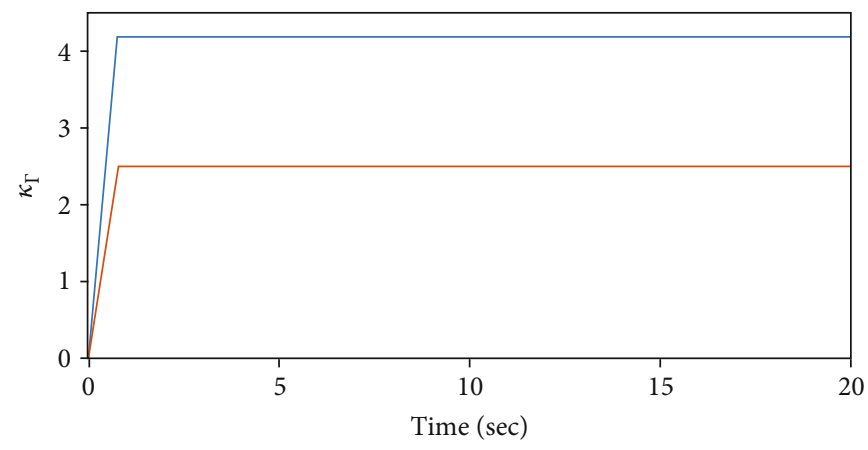

$\begin{aligned}- & \kappa_{\Gamma_{1}} \\ - & \kappa_{\Gamma_{2}}\end{aligned}$

(b)

Figure 2: The curves of estimation errors and adaptive gains of the observer.

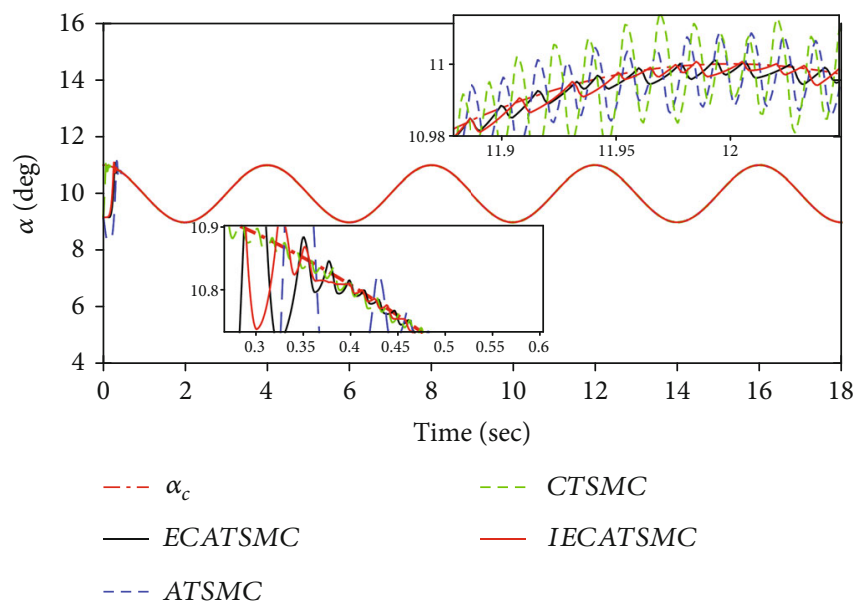

(a)

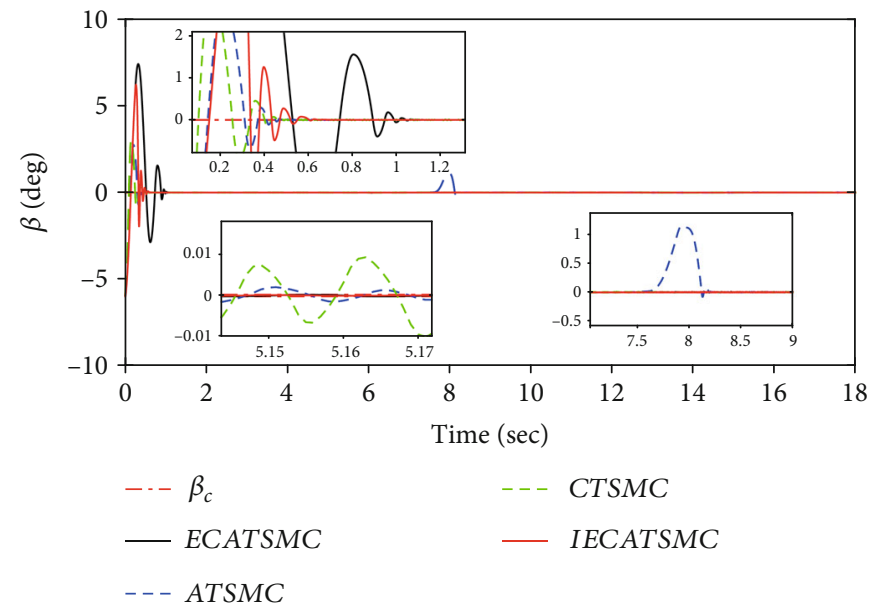

(b)

Figure 3: Tracking effect of yaw and pitch commands.

4.2. Stability Analysis for the Closed-Loop System. In this subsection, the stability of the gliding guided projectile closedloop system (5) is analyzed.

Theorem 7. For the attitude system (5), the adaptive twisting controller is designed as (25), with the adaptive gains defined as $r_{1}=L(t), r_{2}=0.5 L(t)$, where $L(t)$ is obtained from Theorem 4; then, the states $\mathbf{e}_{\Theta}, \mathbf{z}_{\Theta}$ will converge to the origin in finite time.
The proof of Theorem 7 is given in Appendix B.

In order to illustrate the entire control architecture in this paper, the schematic diagram is shown in Figure 1.

\section{Simulation and Results}

To verify the effectiveness of the adaptive algorithm-based controller, the attitude motion of a certain type of GGP 


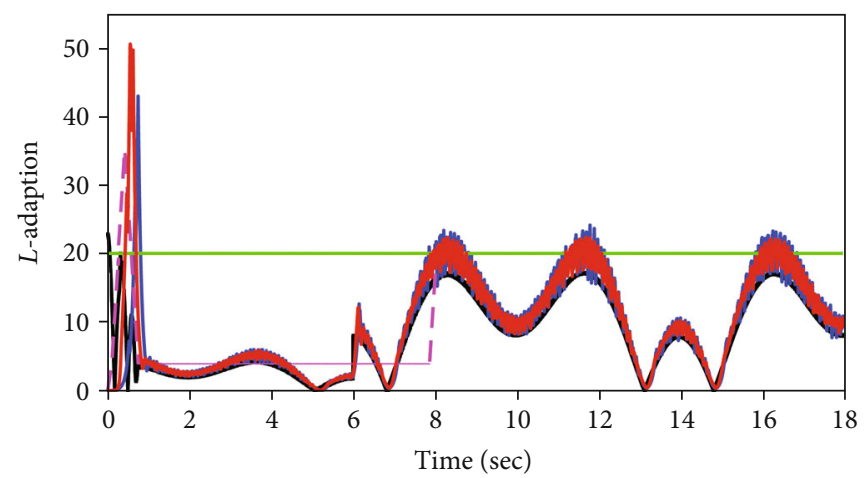

$$
\begin{aligned}
& -2\|\hat{\Delta}\| \quad-\quad L-C T S M C \\
& -L-\text { ECATSMC } \quad L-I E C A T S M C \\
& \text { - - L - ATSMC }
\end{aligned}
$$

Figure 4: Adaptive gain curves.

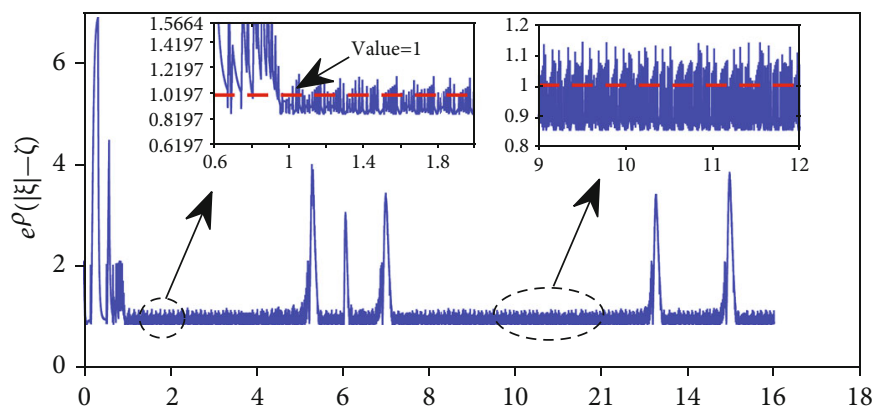

(a)
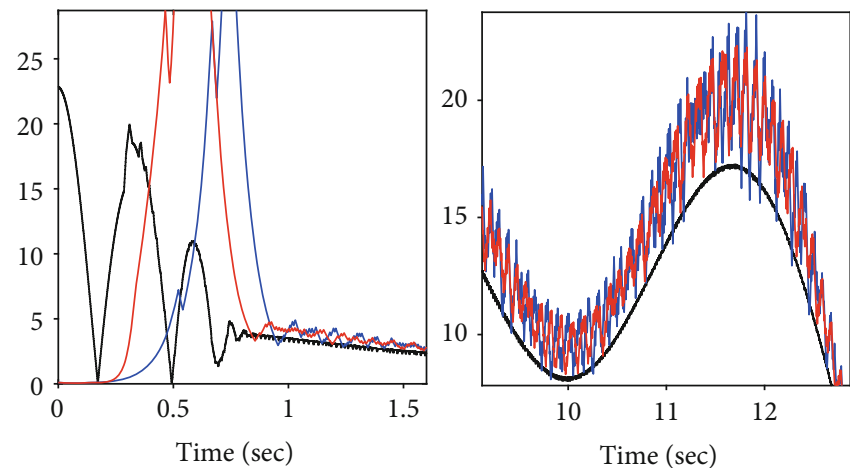

$$
\begin{aligned}
& -2\|\hat{\Delta}\| \\
& -L-E C A T S M C \\
& -L-I E C T A S M C
\end{aligned}
$$

(b)

FIgURE 5: Comparison between IECATSMC and ECATSMC.

is taken as the simulation project. The parameters of the GGP are shown in Table 1. The initial values for the plant are $\mathbf{x}(0)=\left[\begin{array}{llll}0.16 & 0 & -0.1047 & 0\end{array}\right]^{T}$. Reference commands are taken as $\alpha_{c}=\cos (0.5 \pi t)+10 \mathrm{deg}, \beta_{c}=0 \mathrm{deg}$.
To obtain the equivalent control information, we use a low-pass filter formulated as (37) in the simulation, and the parameter is set as $\tau=0.015$. Simulations are performed in MATLAB/simulink environment, where 
sample time is set as $0.001 \mathrm{~s}$, and fourth-order RungeKutta method is chosen as the numerical integration method.

5.1. Comparison. In the simulation, severe disturbances are considered. $\Delta f_{1}, \Delta f_{2}$ are the $40 \%$ uncertainty in $f_{1}, f_{2}$, respectively. $\Delta \mathbf{B}_{1}$ and $\Delta \mathbf{B}_{2}$ are the $10 \%$ uncertainty in $\mathbf{B}_{1}$ and $\mathbf{B}_{2}$, respectively. The external disturbances are

$$
d_{1}=\left\{\begin{array}{l}
9 t-8 \cos \left(\frac{t}{2}\right)-14 \cos \left(\frac{t}{5}\right), \quad 0 \leq t \leq 6 \\
4\left(9 t-8 \cos \left(\frac{t}{2}\right)-14 \cos \left(\frac{t}{5}\right)\right), \quad t>6
\end{array}\right.
$$

$$
d_{2}=\left\{\begin{array}{l}
t+\frac{2}{\pi} \cos \left(\frac{\pi t}{2}\right)-\frac{3}{\pi} \cos \left(\frac{\pi t}{3}\right), \quad 0 \leq t \leq 6, \\
4\left(t+\frac{2}{\pi} \cos \left(\frac{\pi t}{2}\right)-\frac{3}{\pi} \cos \left(\frac{\pi t}{3}\right)\right), \quad t>6 .
\end{array}\right.
$$

For performance comparison, the ATSMC proposed in [34] and CTSMC proposed in [24] are also simulated under the same conditions. The gain adaptive schemes are formulated as

(i) ATSMC [34]: the gain adaptive scheme $\dot{L}$ is defined as

$$
\dot{L}= \begin{cases}\frac{\omega_{1} / \sqrt{2 \gamma_{1}}}{\left(1 / \gamma_{1}\right)-\left(2 L \mathbf{x}_{1}{ }^{T} \mathbf{x}_{1}+\left\|\mathbf{x}_{1}\right\| \mathbf{x}_{2}{ }^{T} \mathbf{x}_{2}\right) /\left(\left(L^{*}-L\right)^{3}\right)} \operatorname{sign}\left(V_{0}-\mu\right), & \text { if } L \geq L_{\min }, \\ \chi, & \text { if } L>L_{\min } .\end{cases}
$$

The term $V_{0}$ can be computed by

$$
V_{0}=L^{2} \mathbf{x}_{\mathbf{1}}{ }^{T} \mathbf{x}_{\mathbf{1}}+\gamma_{1} \frac{\mathbf{x}_{\mathbf{1}}{ }^{T} \mathbf{x}_{\mathbf{2}}}{\left\|\mathbf{x}_{\mathbf{1}}\right\|^{1 / 2}}+L\left\|\mathbf{x}_{\mathbf{1}}\right\| \mathbf{x}_{\mathbf{2}}{ }^{T} \mathbf{x}_{\mathbf{2}}+\frac{1}{4}\left\|\mathbf{x}_{\mathbf{2}}\right\|^{4}
$$

(ii) CTSMC [24]: the gain adaptive scheme $\dot{L}$ is defined as

$$
\dot{L}=0
$$

The parameters of the three methods are listed in Table 2. Besides, to validate the effectiveness of the improved algorithm, the method named ECATSMC whose gain adaptive law is defined as [28]:

$$
\dot{L}=\eta L \operatorname{sign}(\xi)-M\left[L-L^{+}\right]_{+}+M[\mu-L]_{+}
$$

is also simulated, and its control parameters are the same with those of IECATSMC.

Figure 2 shows the curves of the estimation error of $\mathbf{z}_{\Theta}$ and the adaptive gains of the observer $\mathbf{k}_{\Gamma}=\left[\begin{array}{ll}k_{\Gamma_{1}} & k_{\Gamma_{2}}\end{array}\right]^{T}$. From Figure 2(a), we can see that the estimation error can converge to the origin in a short time. Besides, it is shown in Figure 2(b) that the adaptive gains of the observer keep increasing when the estimation error is large; then, they remain constant.

Figure 3 denotes the tracking effect of yaw and pitch commands of the four algorithms. As we can see from Figure 3(a), they both can track the reference commands precisely. After the control begins, CTSMC responses fastest in tracking the commands, IEATSMC takes the second place, and ECATSMC takes the third place, ATSMC responds a little slower than the other three methods. What is more, we can also see from Figure 3(a) that IECATSMC and ECATSMC perform better in terms of tracking accuracy. CTSMC performs a little worse. The tracking effects for pitch command are shown in Figure 3(b). As we see in the picture, ECATSMC responds a little slower than the other three methods. Despite this, ECATSMC can track the command more precisely than CTSMC and ATSMC. Besides, we can also observe that the curve of pith angle under ATSMC deviates from the reference command curve around $t=8 \mathrm{~s}$. This is due to the fact that the intensive disturbance is imposed around that time. On the contrary, the tracking effects of the other three methods do not degrade.

Figure 4 shows the curves of the adaptive gains. We can see from the picture that the gains of IECATSMC and ECATSMC are slightly larger than $2|\widehat{\Delta}|$ in almost the whole control process. It should be noted that this approximation can be easily adjusted by choosing the value of $\varepsilon$. We can also see that ATSMC can increase the gain to satisfy 


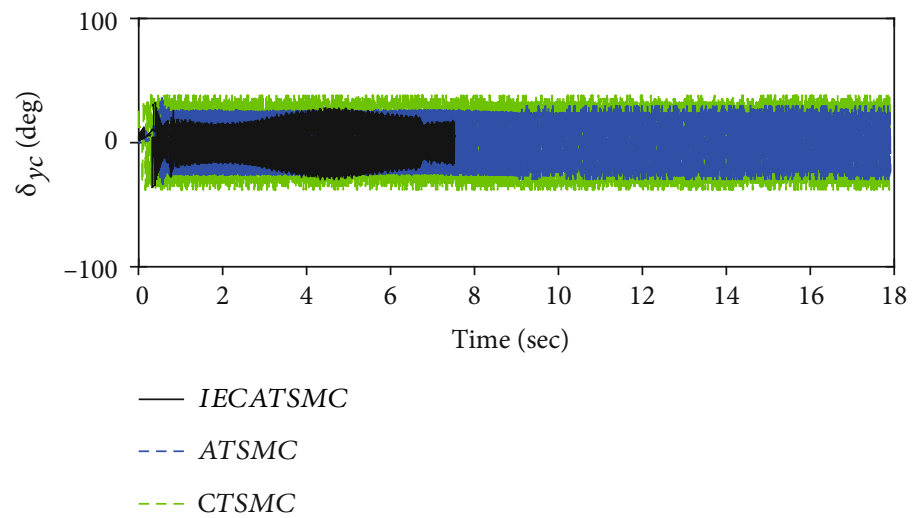

(a)
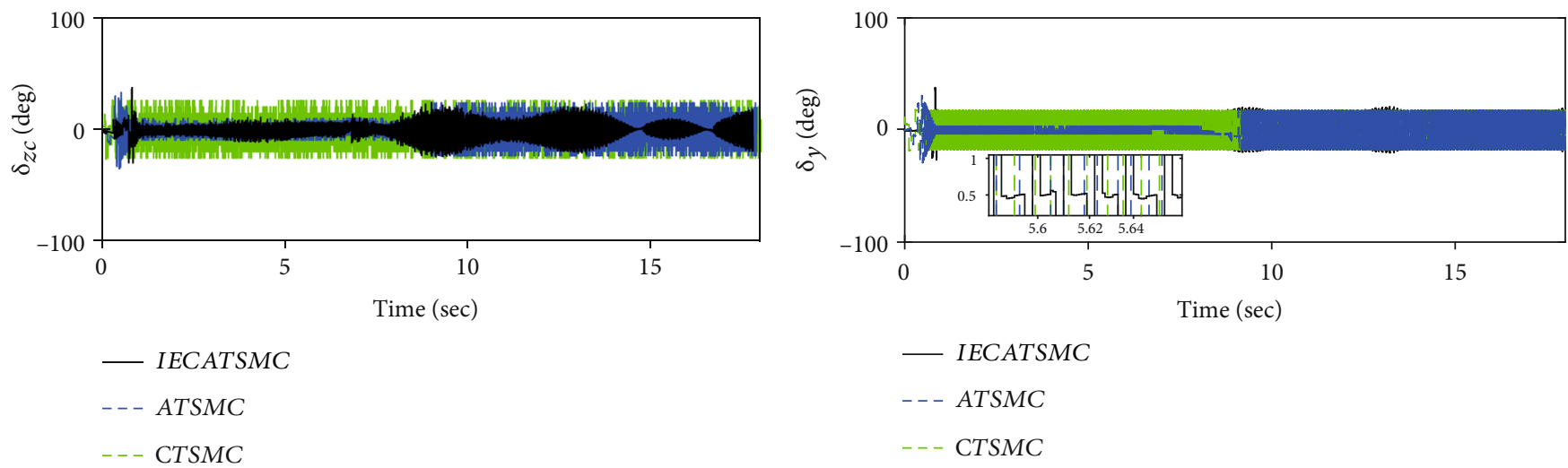

(b)

(c)

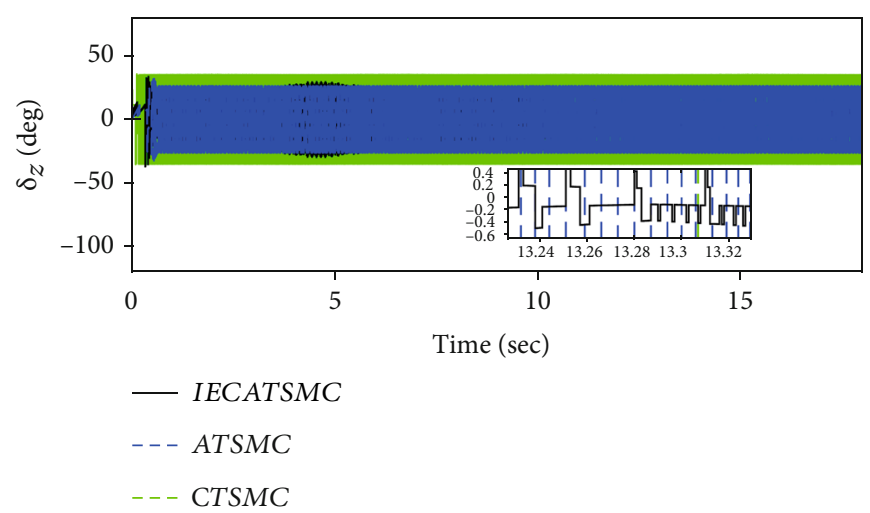

(d)

FIgURE 6: Equivalent canard angle commands.

$L>2\|\widehat{\Delta}\|$ when $2\|\widehat{\Delta}\|$ is larger than $L$. However, in other cases, the gain does not change. Besides, Figure 4 shows that the control gain $L$ under CTSMC keep constant, although it satisfies $L>2\|\widehat{\Delta}\|$, its value is difficult to be set in practical applications given the upper boundary of disturbance is unknown.

Figure 5 shows the comparison between IECATSMC and ECATSMC and the curve of the exponential term. From Figure 5(a), we can see that at the beginning of the control process, the exponential term automatively increases to a large value to accelerate the changing rate of the gain $L$. Actually, when there is a sudden change occurring in $\|\widehat{\Delta}\|$, the exponential term can always adjust its value in time. As a result, IECATSMC reponses faster than ECATSMC, which is demonstrated in Figure 5(b). What is more, when the disturbance changes gently, the exponential term can adjust its value around 1 . The result is that IECATSMC has a smaller amplitude of oscillation than ECATSMC, which enables the former one to seldomly violate the condition of $L>2\|\widehat{\Delta}\|$.

Figure 6 denotes the curves of equivalent canard angle commands and equivalent canard angles. Because the 


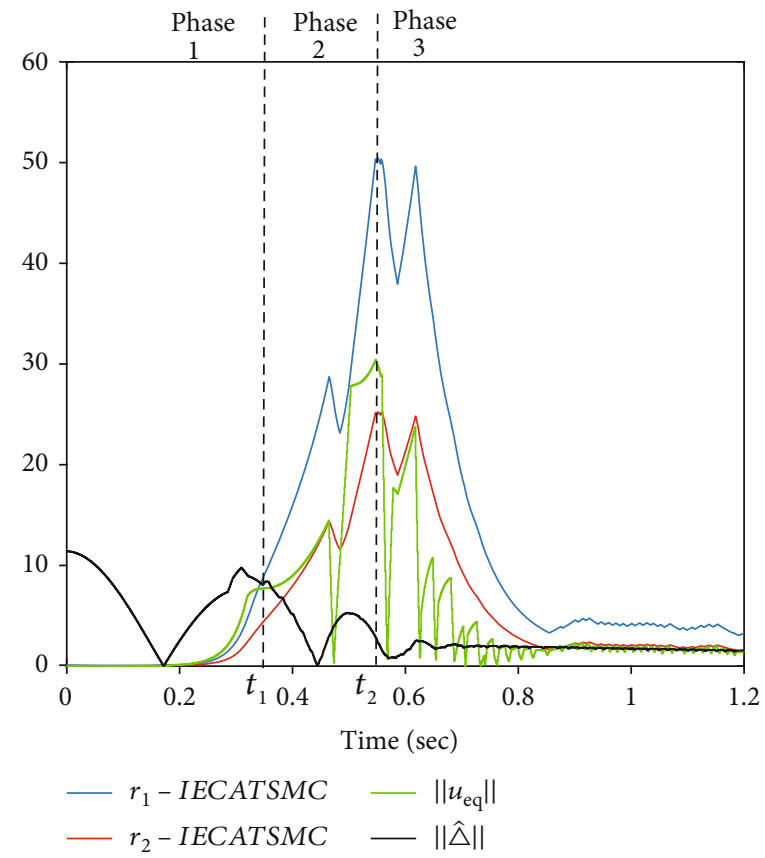

FIgURE 7: Gains in three phases.

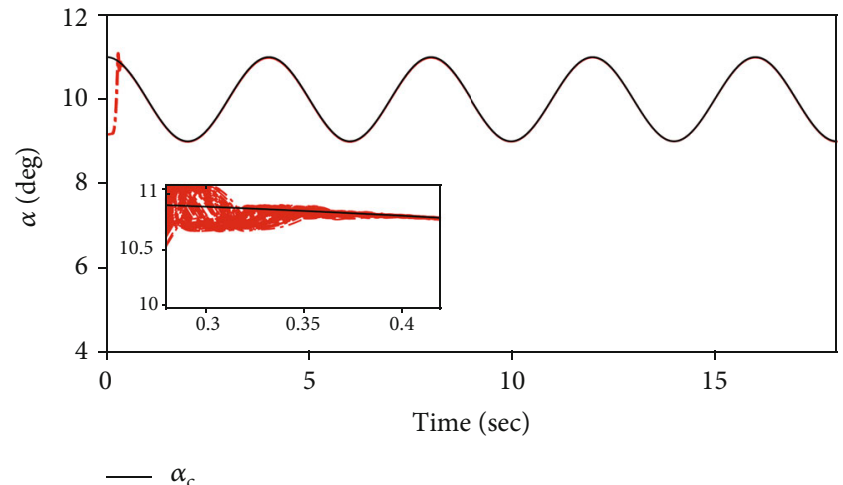

(a)

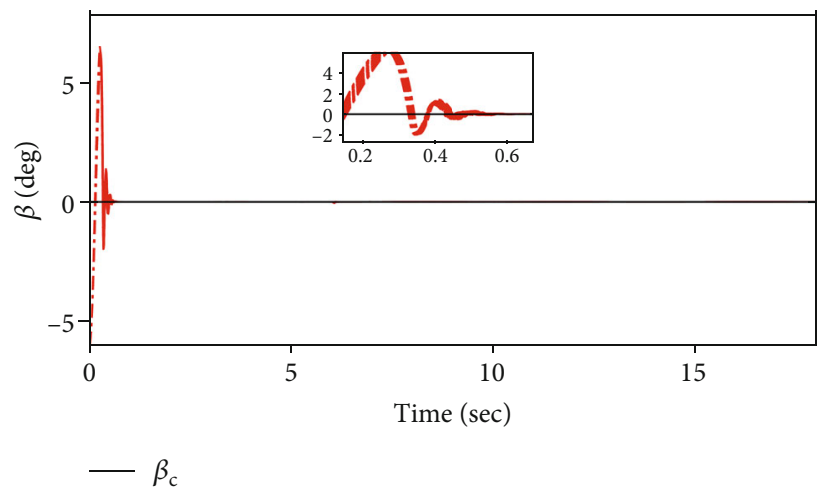

(b)

Figure 8: Tracking effect of yaw and pitch commands.

results of IECATSMC and ECATSMC are very similar, here, we only show the results of the former one for a clear comparison with the other two methods. Because the values of equivalent canard angle commands are closely related with those of the control gains, we can see from Figures 6(a) and 6(b) that IECATSMC greatly attenuates the amplitude of chattering. This is also reflected in Figures 6(c) and 6(d).

Figure 7 shows how the gain is adjusted in the three phases in a clearer manner. As we see from the picture, the gains $r_{1}=L$ and $r_{2}=0.5 L$ continue to increase after control begins, and $L>2\|\widehat{\Delta}\|$ is satisfied after $t>t_{1}$. The gains reach their maximum on $t=t_{2}$. After that, the equivalent control input $\left\|u_{\text {eq }}\right\|$ equals the disturbance; thus, the gains keep decreasing until their curves follow the change of $2\|\widehat{\Delta}\|$. Therefore, Figure 6 verifies the proof.
5.2. Monte Carlo Experiments. In order to further validate the robustness of the proposed control scheme, Monte Carlo experiments are performed 100 times. The external and internal disturbances used in this subsection are the same as that in Section 5.1, and the standard derivations of the errors of the aerodynamic parameters are set to be $30 \%$ of the nominal values.

It can be observed from Figure 8 that the corresponding curves are kept within small envelops, and all of them can track the desired commands accurately.

\section{Conclusion}

In this paper, a robust attitude tracking control scheme is proposed for the gliding-guided projectile. The proposed control method does not require any information on the 
bounds of disturbances. Simulation results show that the proposed method is not only more efficient in chattering attenuation than existing algorithms but also more precise in tracking performance. Besides, in order to make the proposed attitude tracking controller be more robust, actuatorfault situation should be taken into consideration. Therefore, in our future work, we will extend the proposed control scheme with a fault-tolerant approach.

\section{Appendix}

\section{A. Proof of Lemma 3}

Consider the following Lyapunov function candidate [33]:

$$
V_{0}\left(\mathbf{x}_{1}, \mathbf{x}_{2}\right)=L^{2} \mathbf{x}_{1}{ }^{T} \mathbf{x}_{1}+\gamma_{1} \frac{\mathbf{x}_{1}{ }^{T} \mathbf{x}_{2}}{\left\|\mathbf{x}_{1}\right\|^{1 / 2}}+L\left\|\mathbf{x}_{1}\right\| \mathbf{x}_{2}{ }^{T} \mathbf{x}_{2}+\frac{1}{4}\left\|\mathbf{x}_{2}\right\|^{4},
$$

and the above function can be rewritten as

$$
V_{0}\left(\mathbf{x}_{1}, \mathbf{x}_{2}\right)=\left\|\mathbf{x}_{1}\right\| \mathbf{z}^{T} \Gamma \mathbf{z}+\frac{1}{4}\left\|\mathbf{x}_{2}\right\|^{4}
$$

where $\mathbf{z}=\left[\begin{array}{ll}\mathbf{x}_{1} /\left\|\mathbf{x}_{1}\right\|^{1 / 2} & \mathbf{x}_{2}\end{array}\right]^{T}, \boldsymbol{\Gamma}=\left[\begin{array}{cc}L^{2} & \gamma_{1} / 2 \\ \gamma_{1} / 2 & L\end{array}\right]$. To make $\boldsymbol{\Gamma}$ be positive, $\gamma_{1}$ is chosen to satisfy $\gamma_{1}<2 L^{3 / 2}$. By utilizing the relationship $\lambda_{\min }\{\boldsymbol{\Gamma}\}\|\mathbf{z}\|^{2} \leq \mathbf{z}^{T} \Gamma \mathbf{z} \leq \lambda_{\max }\{\boldsymbol{\Gamma}\}\|\mathbf{z}\|^{2}$, we can have

$$
\begin{aligned}
V_{0}\left(\mathbf{x}_{1}, \mathbf{x}_{2}\right)= & \lambda_{\max }\{\boldsymbol{\Gamma}\}\|\mathbf{z}\|^{2}+\frac{1}{4}\left\|\mathbf{x}_{2}\right\|^{4}=\lambda_{\max }\{\boldsymbol{\Gamma}\}\left(\left\|\mathbf{x}_{1}\right\|^{2}+\left\|\mathbf{x}_{1}\right\|\left\|\mathbf{x}_{2}\right\|^{2}\right) \\
& +\frac{1}{4}\left\|\mathbf{x}_{2}\right\|^{4} \leq \frac{3}{2} \lambda_{\max }\{\boldsymbol{\Gamma}\}\left\|\mathbf{x}_{1}\right\|^{2}+\frac{1}{2}\left(\lambda_{\max } \Gamma+\frac{1}{2}\right)\left\|\mathbf{x}_{2}\right\|^{4} \\
= & \rho^{T} P \rho,
\end{aligned}
$$

where $\rho=\left[\begin{array}{ll}\left\|\mathbf{x}_{1}\right\| & \left\|\mathbf{x}_{2}\right\|^{2}\end{array}\right]^{T}$, and the matrix

$$
\mathbf{P}=\left[\begin{array}{cc}
\frac{3}{2} \lambda_{\max }\{\boldsymbol{\Gamma}\} & 0 \\
0 & \frac{1}{2}\left(\lambda_{\max }\{\boldsymbol{\Gamma}\}+\frac{1}{2}\right)
\end{array}\right]
$$

Then, (A.3) can be rewritten as

$$
\begin{aligned}
V_{0}\left(\mathbf{x}_{1}, \mathbf{x}_{2}\right) & \leq \lambda_{\max }\{\mathbf{P}\}\left(\left\|\mathbf{x}_{1}\right\|^{2}+\left\|\mathbf{x}_{1}\right\|\left\|\mathbf{x}_{2}\right\|^{2}\right)+\frac{1}{4}\left\|\mathbf{x}_{2}\right\|^{4} \\
& \leq \lambda_{\max }\{\mathbf{P}\}\left(\left\|\mathbf{x}_{1}\right\|^{\frac{1}{2}}+\left\|\mathbf{x}_{2}\right\|\right)^{4},
\end{aligned}
$$

On the other hand,

$$
\begin{aligned}
V_{0}\left(\mathbf{x}_{1}, \mathbf{x}_{2}\right) & \geq \lambda_{\min }\{\mathbf{P}\}\left(\left\|\mathbf{x}_{1}\right\|^{2}+\left\|\mathbf{x}_{1}\right\|\left\|\mathbf{x}_{2}\right\|^{2}\right)+\frac{1}{4}\left\|\mathbf{x}_{2}\right\|^{4} \\
& \geq \lambda_{\min }\{\boldsymbol{\Gamma}\}\left\|\mathbf{x}_{1}\right\|^{2}+\frac{1}{4}\left\|\mathbf{x}_{2}\right\|^{4}
\end{aligned}
$$

Next, taking the derivative of $V_{0}\left(\mathbf{x}_{1}, \mathbf{x}_{2}\right)$, and substituting (27) into it, yields

$$
\begin{aligned}
\dot{V}_{0}\left(\mathbf{x}_{1}, \mathbf{x}_{2}\right)= & \left(2 L^{2} \mathbf{x}_{1}{ }^{T}+\frac{3}{2} \gamma_{1}\left\|\mathbf{x}_{1}\right\|^{1 / 2} \mathbf{x}_{2}+L \frac{\mathbf{x}_{1}{ }^{T}}{\left\|\mathbf{x}_{1}\right\|}-\mathbf{x}_{2}{ }^{T} \mathbf{x}_{2}\right) \dot{\mathbf{x}}_{1} \\
& +\left(\gamma_{1}\left\|\mathbf{x}_{1}\right\|^{1 / 2} \mathbf{x}_{1}{ }^{T}+2 L\left\|\mathbf{x}_{1}\right\| \mathbf{x}_{2}{ }^{T}+\left\|\mathbf{x}_{2}\right\|^{2} \mathbf{x}_{2}{ }^{T}\right) \dot{\mathbf{x}}_{2} \\
= & -\gamma_{1}\left(L\left\|\mathbf{x}_{1}\right\|^{3 / 2}-\left\|\mathbf{x}_{1}\right\|^{1 / 2} \mathbf{x}_{1}{ }^{T} \mathbf{f}+0.5 L\right. \\
& \left.-\left\|\mathbf{x}_{1}\right\|^{1 / 2}\left\|\mathbf{x}_{2}\right\|^{-1} \mathbf{x}_{1}{ }^{T} \mathbf{x}_{2}\right)-L\left\|\mathbf{x}_{1}\right\|\left(L\left\|\mathbf{x}_{2}\right\|-2 \mathbf{x}_{2}{ }^{T} \mathbf{f}\right) \\
& +\frac{3}{2} \gamma_{1}\left\|\mathbf{x}_{1}\right\|^{1 / 2}\left\|\mathbf{x}_{2}\right\|^{2}-\left(0.5 L\left\|\mathbf{x}_{2}\right\|-\mathbf{x}_{2}{ }^{T} \mathbf{f}\right)\left\|\mathbf{x}_{2}\right\|^{2} \\
\leq & -\gamma_{1}(L-\|\mathbf{f}\|+0.5 L)\left\|\mathbf{x}_{1}\right\|^{3 / 2}-L(L-2\|\mathbf{f}\|)\left\|\mathbf{x}_{1}\right\|\left\|\mathbf{x}_{2}\right\| \\
& +\frac{3}{2} \gamma_{1}\left\|\mathbf{x}_{1}\right\|^{1 / 2}-\left\|\mathbf{x}_{2}\right\|^{2}-\left(0.5 L\left\|\mathbf{x}_{2}\right\|^{-}\|\mathbf{f}\|\right)\left\|\mathbf{x}_{2}\right\|^{2} .
\end{aligned}
$$

Recall that $L>2 D_{0}$ and using the relationship $\|\mathbf{f}\| \leq D_{0}$, we can obtain

$$
\begin{aligned}
\dot{V}_{0}\left(\left\|\mathbf{x}_{1}\right\|,\left\|\mathbf{x}_{2}\right\|\right) \leq & -\left\|\mathbf{x}_{2}\right\|\left(L\left(L-2 D_{0}\right)\left\|\mathbf{x}_{1}\right\|-\frac{3}{2} \gamma_{1}\left\|\mathbf{x}_{1}\right\|^{1 / 2}\left\|\mathbf{x}_{2}\right\|\right) \\
& \left.+\left(\frac{1}{2} L-D_{0}\right)\left\|\mathbf{x}_{2}\right\|^{2}\right)-\gamma_{1}\left(\frac{1}{2} L-D_{0}\right)\left\|\mathbf{x}_{1}\right\|^{3 / 2} \\
= & -\gamma_{1}\left(\frac{1}{2} L-D_{0}\right)\left\|\mathbf{x}_{1}\right\|^{\frac{3}{2}}-\left\|\mathbf{x}_{2}\right\| \varphi^{T} \Psi \varphi,
\end{aligned}
$$

where $\varphi=\left[\left|\mathbf{x}_{1}\right|^{1 / 2}\left|\mathbf{x}_{2}\right|\right]^{T}$, and the matrix $\boldsymbol{\Phi}$ is

$$
\Psi=\left[\begin{array}{cc}
2 L\left(\frac{1}{2} L-D_{0}\right) & -\frac{3}{4} L \\
-\frac{3}{4} L & \frac{1}{2} L-D_{0}
\end{array}\right],
$$

If $L>2 D_{0}, 0<\gamma_{1}<(4 \sqrt{2}) / 3 \sqrt{L}\left(0.5 L-D_{0}\right)$ holds, then the matrix $\Psi$ is positive definite, which implies $\dot{V}_{0}\left(\mathbf{x}_{1}, \mathbf{x}_{2}\right)$ is negative definite, and the following inequality holds

$$
\lambda_{\max }(\Psi)\left(\left\|\mathbf{x}_{1}\right\|+\left\|\mathbf{x}_{2}\right\|^{2}\right) \leq \varphi^{T} \Psi \varphi \leq \lambda_{\max }(\Psi)\left(\left\|\mathbf{x}_{1}\right\|+\left\|\mathbf{x}_{2}\right\|^{2}\right) .
$$

By using the following well-known inequality,

$$
\left(|x|^{p}+|y|^{p}\right)^{1 / q} \leq 2^{(1 / q-1 / p)}\left(|x|^{p}+|y|^{p}\right)^{1 / p},
$$


(A.8) can be rewritten as

$$
\begin{aligned}
\dot{V}_{0}\left(\left\|\mathbf{x}_{1}\right\|,\left\|\mathbf{x}_{2}\right\|\right) & \leq-\gamma_{1}\left(\frac{1}{2} L-D_{p 0}\right)\left\|\mathbf{x}_{1}\right\|^{3 / 2}-\left\|\mathbf{x}_{2}\right\|-\lambda_{\min }(\Psi)\left(\left\|\mathbf{x}_{1}\right\|+\left\|\mathbf{x}_{2}\right\|^{2}\right) \\
& \leq-\gamma_{1}\left(\frac{1}{2} L-D_{p 0}\right)\left\|\mathbf{x}_{1}\right\|^{3 / 2}-\left\|\mathbf{x}_{2}\right\|^{3}-\lambda_{\min }(\Psi) \\
& \leq-E\left(\left\|\mathbf{x}_{1}\right\|^{3 / 2}+\left\|\mathbf{x}_{2}\right\|^{3}\right) \leq-\frac{E}{2^{2 / 3}}\left(\left\|\mathbf{x}_{1}\right\|^{1 / 2}+\left\|\mathbf{x}_{2}\right\|\right)^{3} \\
& \leq-\frac{E}{2^{2 / 3} \lambda_{\max }^{3 / 4}(\mathbf{P})} V_{0}^{\frac{3}{4}}\left(\left\|\mathbf{x}_{1}\right\|,\left\|\mathbf{x}_{2}\right\|\right),
\end{aligned}
$$

where $E=\min \left\{\gamma_{1}\left((1 / 2) L-D_{0}\right), \lambda_{\min }(\Psi)\right\}$. Let us denote the upper bound of $\lambda_{\max }(\mathbf{P})$ as $\bar{\lambda}_{\max }(\mathbf{P})$, the lower bound of $E$ as $\underline{E}$, then (A.10) can be modified as

$$
\begin{aligned}
\dot{V}_{0}\left(\mathbf{x}_{1}, \mathbf{x}_{2}\right) & \leq-\frac{E}{2^{2 / 3} \lambda_{\max }^{3 / 4}(\mathbf{P})} V_{0}^{3 / 4}\left(\mathbf{x}_{1}, \mathbf{x}_{2}\right) \\
& \leq-\frac{\underline{E}}{2^{2 / 3} \bar{\lambda}_{\max }^{3 / 4}(\mathbf{P})} V_{0}^{3 / 4}\left(\mathbf{x}_{1}, \mathbf{x}_{2}\right) .
\end{aligned}
$$

Therefore, the states $\mathbf{x}_{1}$ and $\mathbf{x}_{2}$ will converge to origin in finite time at most $t_{2} \leq t_{0}+t_{1}+\left(16 \bar{\lambda}_{\max }^{3 / 4}(\mathbf{P}) V_{0}^{1 / 4}\left(t_{1}\right)\right) / \bar{E}$.

\section{B. Proof of Theorem 7}

The proof can be divided into two steps. Firstly, it will be demonstrated that the closed-loop system (26) is bounded with the existence of estimation error e. Next, the trajectories of the closed loop (26) without estimation error will be analyzed. Since the uncertainty $\widehat{\Delta}$ is bounded due to the boundedness of $\dot{\mathbf{e}}$, there exists a positive constant $\widehat{C}$, which satisfies $\|\widehat{\Delta}\| \leq C<\widehat{C}$.

Choose a Lyapunov function candidate as $\widehat{V}_{0}\left(\mathbf{e}_{\Theta}, \mathbf{z}_{\Theta}\right)=$ $V_{0}\left(\mathbf{e}_{\Theta}, \widehat{\mathbf{z}}_{\Theta}\right)=L^{2} \mathbf{e}_{\Theta}^{2}+\gamma_{1}\left|\mathbf{e}_{\Theta}\right|^{3 / 2} \widehat{\mathbf{z}}_{\Theta}$ $\operatorname{sign}\left(\mathbf{e}_{\Theta}\right)+L\left|\mathbf{e}_{\Theta}\right| \mathbf{z} \wedge_{\Theta}{ }^{2}+(1 / 4) \mathbf{z} \wedge_{\Theta}{ }^{4}$. According to the analysis in phases 1 and 2 in Theorem 4 , the gain $L(t)$ keeps increasing until $L(t)>2 \widehat{C}$, and the flowing inequality can hold

$$
\dot{\hat{V}}_{0}\left(\mathbf{e}_{\Theta}, \widehat{\mathbf{z}}_{\Theta}\right) \leq-\frac{E}{2^{2 / 3} \lambda_{\max }^{3 / 4}(\mathbf{P})} V_{0}^{3 / 4}\left(\mathbf{x}_{1}, \mathbf{x}_{2}\right)+\Lambda,
$$

where

$$
\Lambda=-2 L^{2} \mathbf{e}_{\Theta}^{T} \mathbf{e}-\frac{3}{2} \gamma_{1} \| \frac{\mathbf{e}_{\Theta} \|^{1}}{2 \mathbf{z} \wedge_{\Theta}^{T} \mathbf{e}}-L \mathbf{z} \wedge_{\Theta}{ }^{T} \widehat{\mathbf{z}}_{\Theta} \frac{\mathbf{e}_{\Theta}{ }^{T}}{\left\|\mathbf{e}_{\Theta}\right\|} \mathbf{e} .
$$

Note that the estimation error e is bounded, assume $\|\mathbf{e}\| \leq H$, where $H$ is a positive constant. Then, we obtain

$$
\Lambda \leq H \Xi^{T} R \Xi \leq H \lambda_{\max }(\mathbf{R})\left(\left\|\mathbf{e}_{\Theta}\right\|+\left\|\widehat{\mathbf{z}}_{\Theta}\right\|^{2}\right),
$$

where $\boldsymbol{\Xi}=\left[\left\|\mathbf{e}_{\Theta}\right\|^{1 / 2},\left\|\mathbf{z} \wedge_{\Theta}\right\|\right]^{T}$, and the matrix $\mathbf{R}=$ $\left[\begin{array}{cc}2 L^{2} & (1 / 2) \gamma_{1} \\ (1 / 3) \gamma_{1} & L\end{array}\right]$, which is positive definite according to the value of $\gamma_{1}$. From (A.5), we can verify that $\widehat{V}_{0} \geq \lambda_{\text {min }}$ $(\Gamma)-\left\|\mathbf{e}_{\Theta}\right\|^{2}+1 / 4\left\|\widehat{\mathbf{z}}_{\Theta}\right\|^{4} \geq \underline{\lambda}\left(\left\|\mathbf{e}_{\Theta}\right\|^{2}+1 / 4\left\|\widehat{\mathbf{z}}_{\Theta}\right\|^{4}\right)$ where $\underline{\lambda}=$ $\min \left\{\lambda_{\min }(\boldsymbol{\Gamma}), 1 / 4\right\}$. Therefore,

$$
\begin{aligned}
\Lambda & \leq H \lambda_{\max }(\mathbf{R})\left(\left\|\mathbf{e}_{\Theta}\right\|+\left\|\widehat{\mathbf{z}}_{\Theta}\right\|^{2}\right), \leq H \lambda_{\max }(\mathbf{R}) \sqrt{2\left\|\mathbf{e}_{\Theta}\right\|^{2}+\left\|\widehat{\mathbf{z}}_{\Theta}\right\|^{4}}, \\
& \leq \frac{\sqrt{2} H \lambda_{\max }(\mathbf{R})}{\sqrt{\underline{\lambda}}} V_{0}^{1 / 2} .
\end{aligned}
$$

Using (B.1) along with the above inequality, one has

$$
\begin{aligned}
\dot{\hat{V}}_{0} & \leq-\frac{E}{4 \lambda_{\max }^{3 / 4}(\mathbf{P})} \widehat{V}_{0}^{3 / 4}+\frac{\sqrt{2} H \lambda_{\max }(\mathbf{R})}{\sqrt{\underline{\lambda}}} \widehat{V}_{0}^{1 / 2}, \\
& =-\frac{E}{4 \lambda_{\max }^{3 / 4}(\mathbf{P})} V_{0}^{1 / 2}\left(\widehat{V}_{0}^{1 / 4}-\frac{4 \sqrt{2} H \lambda_{\max }(\mathbf{R}) \lambda_{\max }(\mathbf{P})^{3 / 4}}{\sqrt{\underline{\lambda}} E}\right) .
\end{aligned}
$$

Therefore, it is easy to verify that $\widehat{V}_{0}$ will be confined in a region which is a little larger than $C_{V}=\left\{\widehat{V}_{0}\right.$ $\left.\left.\leq\left(4 \sqrt{2} H \lambda_{\max }(\mathbf{R})\right)^{4} \lambda_{\max }(\mathbf{P})^{3}\right) / \underline{\lambda}^{2} E^{4}\right\}$. Thus, $\widehat{V}_{0}$ is bounded, which means the states $\mathbf{e}_{\Theta}$ and $\widehat{\mathbf{z}}_{\Theta}$ are bounded. In Section 3 , we have shown that the estimation error $\mathbf{e}$ for the state $\mathbf{z}_{\Theta}$ will converge to zero in finite time. Without loss of generality, the convergence time is set as $T_{o}$. So, $\widehat{\mathbf{z}}_{\Theta}=\mathbf{z}_{\Theta}$ holds, when $t>T_{o}$. Thus, the closed-loop system (26) is reduced to

$$
\left(\begin{array}{l}
\dot{\mathbf{e}}_{\Theta}=\mathbf{z}_{\Theta}, \\
\dot{\mathbf{z}}_{\Theta}=-r_{1} \frac{\mathbf{e}_{\Theta}}{\left\|\mathbf{e}_{\Theta}\right\|}-r_{2} \frac{\widehat{\mathbf{z}}_{\Theta}}{\left\|\widehat{\mathbf{z}}_{\Theta}\right\|}+\Delta,
\end{array}\right.
$$

which is identical to (27). By following the analysis in Theorem 4 , we can conclude that the attitude tracking error $\mathbf{e}_{\Theta}$ will converge to zero at most $t_{z} \leq T_{o}+t_{1}+\left(16 \bar{\lambda}_{\max }^{3 / 4}(\mathbf{P}) V_{0}^{1 / 4}\left(t_{1}\right)\right) /$ $\bar{E}$. What is more, the minimum possible value of the gain $L$ can be kept in phase 3, which attenuates the amplitude of chattering.

\section{Nomenclature}

$\alpha, \beta: \quad$ Angle of attack, side slip angle

$\theta, \varphi$ : Inclination angle, and deflection angle of flight trajectory

Ү, $\phi: \quad$ Pitch angle and yaw angle

$\delta_{z}, \delta_{y}$ : Deflection angle of canards

$m$ : $\quad$ Mass of the projectile

$v$ : $\quad$ Speed of the projectile

Q: $\quad$ Dynamic pressure

D: $\quad$ Projectile diameter 
E, C: Equatorial damping coefficient and polar damping coefficient [10]

$l, s: \quad$ Characteristic length and characteristic area

$m_{z}^{\prime}$ : The derivation of the static moment

$m_{z z}^{\prime}$ : The derivation of equatorial damping moment

$m_{\sigma}^{\prime}: \quad$ The derivation of control moment generated by canard

$C_{L}^{\alpha}, C_{L}^{\delta}$ : Derivations of the lift induced by projectile body and canard

$m_{\mu}, m_{\mu}^{\prime \prime}$ Magnus force and the derivation of Magnus moment.

\section{Data Availability}

The data used to support the findings of this study are available from the corresponding author upon request.

\section{Conflicts of Interest}

The authors declare that there is no conflict of interest regarding the publication of this paper.

\section{References}

[1] K. Rakesh, "Modified cubic b-spline quasi-interpolation numerical scheme for hyperbolic conservation laws," Applicable Analysis, vol. 226, no. 10, pp. 1-22, 2018.

[2] G. Strub, S. Dobre, V. Gassmann, S. Theodoulis, and M. Basset, "Pitch-Axis identification for a guided projectile using a WindTunnel-Based experimental setup," IEEE/ASME Transactions on Mechatronics, vol. 21, no. 3, pp. 1357-1365, 2016.

[3] Q. Chen and Z. Y. Wang, "Optimal trajectory design under uncertainty for a gliding guided projectile," Acta Aeronautica ET Astronautica Sinica, vol. 35, no. 9, pp. 2593-2604, 2014.

[4] R. G. Yang, M. W. Sun, and Z. Q. Chen, "ARDC-based attitude control optimization and simulation," Journal of System Simulation, vol. 40, no. 11, pp. 2689-2693, 2010.

[5] B. Tian, L. Yin, and W. Hong, "Finite-Time reentry attitude control based on adaptive multivariable disturbance compensation," IEEE Transactions on Industrial Electronics, vol. 62, no. 9, pp. 5889-5898, 2015.

[6] W. Koch, R. Mancuso, R. West, and A. Bestavros, "Reinforcement learning for uav attitude control," ACM Transactions on Cyber-Physical Systems, vol. 3, no. 2, pp. 5889-5898, 2019.

[7] B. Xu, D. Wang, and Y. Zhang, "DOB-Based neural control of flexible hypersonic flight vehicle considering wind effects," IEEE Transactions on Industrial Electronics, vol. 64, no. 11, pp. 8676-8685, 2017.

[8] H. Yang, L. Cheng, Y. Xia, and Y. Yuan, "Active disturbance rejection attitude control for a dual closed-loop quadrotor under gust wind," IEEE Transactions on Control Systems Technology, vol. 26, no. 4, pp. 1400-1405, 2018.

[9] J. Liu, H. An, Y. Gao, C. Wang, and L. Wu, "Adaptive control of hypersonic flight vehicles with limited angle-of-attack," IEEE/ASME Transactions on Mechatronics, vol. 23, no. 2, pp. 883-894, 2018.

[10] S. Xu, G. Sun, and W. Sun, "Fuzzy logic based fault-tolerant attitude control for nonlinear flexible spacecraft with sampled-data input," Journal of the Franklin Institute, vol. 354 , no. 5, pp. 2125-2156, 2017.
[11] M. A. Zou, K. D. Kumar, Z. G. Hou, and X. Liu, "Finite-time attitude tracking control for spacecraft using terminal sliding mode and chebyshev neural network," IEEE Transactions on Systems, Man and Cybernetics, Part B (Cybernetics), vol. 41, no. 4, pp. 950-963, 2011.

[12] M. J. Zheng and S. J. Xu, "Backstepping control for attitude control system of an underactuated spacecraft," Journal of Astronautic, vol. 27, no. 5, pp. 947-951, 2006.

[13] O. Eldad, E. G. Lightsey, and C. Claudel, "Minimum-time attitude control of deformable solar sails with model uncertainty," Journal of Spacecraft and Rockets, vol. 54, no. 4, pp. 1-8, 2017.

[14] N. M. Suhadis and R. Varatharajoo, "Passive attitude control torque generation performances of a gravity gradient stabilized satellite," Applied Mechanics and Materials, vol. 225, no. 8, pp. 458-463, 2018.

[15] X. Hu, B. Xu, and C. Hu, "Robust adaptive fuzzy control for HFV with parameter uncertainty and unmodeled dynamics," IEEE Transactions on Industrial Electronics, vol. 65, no. 11, pp. 8851-8860, 2018.

[16] W. G. Zhang and W. J. Yi, “Adaptive gliding-guided projectile attitude tracking controller design based on RBF neuro-sliding mode technique," International Journal of Aeronautical and Space Sciences, vol. 21, pp. 504-512, 2019.

[17] S. Mobayen, M. J. Yazdanpanah, and V. J. Majd, “A finite-time tracker for nonholonomic systems using recursive singularityfree FTSM," in Proceedings of the 2011 American Control Conference, pp. 1720-1725, San Francisco, CA, 2011.

[18] S. Mobayen and F. Tchier, "Nonsingular fast terminal sliding mode stabilizer for a class of uncertain nonlinear systems based on disturbance observer," Entia Iranica, vol. 24, no. 3, 2017.

[19] S. Mobayen, "Design of LMI-based sliding mode controller with an exponential policy for a class of underactuated systems," Complexity, vol. 21, no. 5, 124 pages, 2016.

[20] S. Mobayen, "Robust finite-time composite nonlinear feedback control for synchronization of uncertain chaotic systems with nonlinearity and time-delay," Chaos Solitons \& Fractals, vol. 114, pp. 46-54, 2018.

[21] H. Prathap, V. Brinda, and S. Ushakumari, "Robust flight control of a typical RLV during re-entry phase," in 2013 IEEE International Conference on Control Applications (CCA), pp. 716-721, Hyderabad, 2013.

[22] J. Reiner, G. J. Balas, and W. L. Garrard, "Flight control design using robust dynamic inversion and time-scale separation," Automatica, vol. 32, no. 11, pp. 1493-1504, 1996.

[23] S. Bharadwaj, "Entry trajectory tracking law via feedback linearization," Journal of Guidance, Control, and Dynamics, vol. 21, no. 5, pp. 726-732, 2012.

[24] A. Levant, "Sliding order and sliding accuracy in sliding mode control," International Journal of Control, vol. 58, no. 6, pp. 1247-1263, 1993.

[25] D. Qi, Q. Zong, B. Tian, and W. Fang, "Adaptive-gain multivariable super-twisting sliding mode control for reentry RLV with torque perturbation," International Journal of Robust and Nonlinear Control, vol. 27, no. 4, pp. 620-638, 2017.

[26] V. Hoang, M. D. Phung, and Q. Ha, “Adaptive twisting sliding mode control for quadrotor unmanned aerial vehicles," in 2017 11th Asian Control Conference (ASCC), pp. 671-676, Gold Coast, QLD, Australia, 2017.

[27] C. Negrete, Y. Daniel, and J. A. Moreno, "Second-order sliding mode output feedback controller with adaptation," 
International Journal of Adaptive Control and Signal Processing, vol. 30, no. 8-10, pp. 1523-1543, 2016.

[28] V. I. Utkin and A. S. Poznyak, "Adaptive sliding mode control with application to super-twist algorithm: equivalent control method," Automatica, vol. 49, no. 1, pp. 39-47, 2013.

[29] Q. Dong, Q. Zong, and B. Tian, "Adaptive disturbance observer-based finite-time continuous fault-tolerant control for reentry RLV," International Journal of Robust and Nonlinear Control, vol. 27, no. 18, pp. 4275-4295, 2017.

[30] Q. P. Xu, X. G. Wang, and Z. Y. Wang, "Design of attitude decoupling controller for gliding guided projectile based on active disturbance rejection control," Systems Engineering and Electronics, vol. 40, no. 2, pp. 384-392, 2018.

[31] X. F. Qian, R. X. Lin, and Y. N. Zhao, Missile Flight Aerodynamics, Beijing institute of technology press, Beijing, China, 2008.

[32] X. Yan, S. Yang, and C. Zhang, "Coning motion of spinning missiles induced by the rate loop," Journal of Guidance Control \& Dynamics, vol. 33, no. 5, pp. 1490-1499, 2010.

[33] Z. Guo, J. Chang, J. Guo, and J. Zhou, “Adaptive twisting sliding mode algorithm for hypersonic reentry vehicle attitude control based on finite-time observer," ISA Transactions, vol. 77, pp. 20-29, 2018.

[34] Y. B. Shtessel, J. A. Moreno, and L. M. Fridman, “Twisting sliding mode control with adaptation: Lyapunov design, methodology and application," Automatica, vol. 75, no. 1, pp. 229-235, 2017. 